\title{
Circulating microRNAs as biomarkers for early detection of diffuse-type gastric cancer using a mouse model
}

\author{
P Rotkrua ${ }^{1,3,4}$, S Shimada ${ }^{1,3}, \mathrm{~K}$ Mogushi $^{2}$, Y Akiyama ${ }^{1}, \mathrm{H}$ Tanaka ${ }^{2}$ and Y Yuasa ${ }^{*, 1}$ \\ ${ }^{1}$ Division of Molecular Oncology, Graduate School of Medical and Dental Sciences, Tokyo Medical and Dental University, 1-5-45 \\ Yushima, Bunkyo-ku, Tokyo 113-8519, Japan and 2Division of Systems Biology, Graduate School of Biomedical Science, Tokyo \\ Medical and Dental University, 1-5-45 Yushima, Bunkyo-ku, Tokyo 113-8519, Japan
}

Background: Diffuse-type gastric cancer (DGC) exhibits rapid disease progression and a poor prognosis. There are no effective serum biomarkers for early detection of DGC. We have established an E-cadherin/p53 double conditional knockout (DCKO) mouse line that recapitulates human DGC morphologically and molecularly. In this study we tried to identify circulating microRNAs (miRNAs) as non-invasive biomarkers for DGC diagnosis using DCKO mice.

Methods: We performed miRNA microarray and quantitative reverse transcription-PCR analyses of tissue and serum samples from DCKO mice with DGC and age-matched littermate controls.

Results: Comparative analyses showed that mouse and human primary gastric cancers have similar miRNA expression patterns. Next, we selected some candidate miRNAs highly expressed in sera and cancer tissues of DCKO mice for further evaluation. TaqMan quantitative RT-PCR analyses indicated that four of them, miR-103, miR-107, miR-194 and miR-210, were significantly upregulated in sera of both early and advanced-stage DGC-bearing mice compared with in corresponding controls. Receiveroperating characteristic curve analyses demonstrated that these four miRNAs can discriminate DGC-positive cases from normal ones with high sensitivity and specificity.

Conclusion: These observations suggest that this mouse model of DGC is useful for identifying serum biomarkers, and we found circulating miRNAs that can accurately detect DGC at an early stage.

Gastric cancer (GC) is the fourth most common cancer and the second leading cause of cancer death worldwide (Ferlay et al, 2010). Gastric cancer can be classified into two major subtypes: intestinal-type GC (IGC) and diffuse-type GC (DGC) (Laurén, 1965; Japanese Gastric Cancer Association, 1998; Yuasa, 2003). Diagnosis of GC is frequently missed or delayed because there are no symptoms or signs in early stages of the disease. Although endoscopic and microscopic examinations exhibit high reliability for early detection of GC, they are invasive and inappropriate for routine screening. Routine clinical application requires easily implementable tests for biomarker analyses. Some markers in serum such as CEA and CA19-9 are not sensitive or specific enough (Liu et al, 2011). Thus, the best way to use serum tumour markers for diagnosing GC has not been found.

MicroRNAs (miRNAs), which are non-coding RNAs of $\sim 22$ nucleotides in length, inhibit gene expression at the posttranscriptional level, and have crucial roles in a broad range of physiological and pathological processes. MicroRNAs are

\footnotetext{
*Correspondence: Dr Y Yuasa; E-mail: yuasa.monc@tmd.ac.jp

${ }^{3}$ These authors contributed equally to this work.

${ }^{4}$ Present address: Department of Preclinical Science (Biochemistry), Faculty of Medicine, Thammasat University, Klong Luang, Pathumthani 12121, Thailand.
} 
estimated to regulate the expression of $>60 \%$ of human protein-coding genes. Surprisingly, miRNAs show unexpected stability in serum. There are at least three proposed mechanisms underlying how miRNAs are released and protected in the bloodstream. The delivery of miRNAs is mediated by exosome and microvesicle (Valadi et al, 2007), Argonaute2 (Ago2) (Arroyo et al, 2011), and high-density lipoprotein (Vickers et al, 2011). Several lines of evidence have demonstrated that circulating miRNAs exhibit unique profiles for each tumour and histopathological subtype (Cortez et al, 2011; Song and Meltzer, 2012). Therefore, the discovery of miRNAs in serum opens up the possibility of novel biomarkers for non-invasive diagnostic screening.

Even though some circulating miRNAs have recently been identified as potential biomarkers for several types of cancers including GC, the results were inconsistent among studies. This might be due to differences in the histopathological subtypes of the study subjects, or the lack of standardised sample collection and storage methods. The utilisation of genetically engineered mouse models could minimise these problems because we can control the age of the mice, the stage of the tumours, homogeneous breeding, environmental factors, the health conditions and the sampling protocol (Kuick et al, 2007). We have established an E-cadherin/ p53 double conditional knockout (DCKO) mouse model that recapitulates human DGC morphologically and molecularly (Shimada et al, 2012). Diffuse-type gastric cancers develop from the age of 6 months in DCKO mice, and early- and advanced-stage DGCs are found in $100 \%$ and $69 \%$ of 12-month-old ones, respectively. Moreover, lymph node (LN) metastasis of DGC was observed in $40 \%$ of cancer-positive cases.

In this study, first, we demonstrated that DCKO mouse and human GCs are very similar in their miRNA expression patterns. Next, we searched for candidate DGC-specific miRNA biomarkers in sera using DCKO mice at different ages, that is, with each stage of DGC. Our results suggested that miR-103, miR-107, miR-194 and miR-210 were significantly upregulated in both DCKO mouse sera and DGC tissues compared with in control mice, and that these miRNA signatures are useful biomarkers for detecting earlystage DGC.

\section{MATERIALS AND METHODS}

Mouse tissue and serum samples. The DCKO (Atp $4 b-\mathrm{Cre}^{+}$; $\left.C d h 1^{\text {loxP/loxP }} ; \operatorname{Trp} 53^{\text {loxP/loxP }}\right)$ mouse line was described previously (Shimada et al, 2012). To minimise genetic background differences, Atp $4 b-C r e^{-} ; C d h 1^{\text {loxP/loxP }} ; \operatorname{Trp} 53^{\text {loxP/loxP }}$ age-matched littermates were used as controls. Tumour tissues from DCKO mice and normal tissues from control mice were dissected out, and subsequently formalin-fixed and paraffin-embedded for histological analyses. Pathological classification was performed according to the criteria established by the Japanese Gastric Cancer Association (Japanese Gastric Cancer Association, 1998) and Laurén's classification (Laurén, 1965). Whole blood was taken by cardiac puncture from mice at different ages (3,6-12 and 12 months). The blood samples were allowed to stand at room temperature for at least $1 \mathrm{~h}$ to a maximum of $2 \mathrm{~h}$. Mouse sera were separated from clots by centrifugation at 15000 r.p.m. for $10 \mathrm{~min}$ at $4{ }^{\circ} \mathrm{C}$, and stored at $-80^{\circ} \mathrm{C}$. All animal experiments were conducted following the protocols approved by the Institutional Animal Care and Use Committee of Tokyo Medical and Dental University.

Microarray profiling of tissue and serum miRNAs. Total RNA was extracted from primary GC and LN metastases of three DCKO mice, and normal stomach and LN of three age-matched littermates with TRIzol reagent (Invitrogen, Carlsbad, CA, USA).
MicroRNA microarray analyses were carried out by an outsource research company, Toray Industries, Inc. (Kanagawa, Japan). MicroRNA microarray profiling was performed on Mouse miRNA Oligo chip ver.16. The nucleotides on the chip can detect $\sim 1100$ types of mouse miRNAs selected from database miRBase (http:// www.mirbase.org/) release 16.0.

To assess contamination by normal tissues of tumour samples, we performed single-stranded cDNA synthesis using SuperScript III reverse transcriptase (Invitrogen) and RT-PCR with the primer sets shown in Supplementary Table S1. As mentioned in our previous report (Shimada et al, 2012), Fibronectin 1 (Fn1) and Atp $4 a$ are good markers for distinguishing GC from normal gastric mucosae in DCKO mice (Supplementary Figure S1A). Mucin 1 $(\mathrm{Mucl})$ and $\mathrm{Cd} 8 \mathrm{a}$ are tissue-specific markers of GC and normal LN, respectively, (Supplementary Figure S1B). Transcripts of Gapdh, glyceraldehyde-3-phosphate dehydrogenase, and Hmbs, a house keeping gene coding porphobilinogen deaminase, were amplified as internal controls.

One hundred microliters of each serum sample from five DCKO mice (12 months old) and five age-matched control mice were pooled to give a final volume of $500 \mu \mathrm{l}$. MicroRNA was extracted from the pooled sera based on Toray's original protocol. RNA quality and concentration were assessed by this company using an Agilent 2100 Bioanalyzer and a spectrophotometer (Agilent Technologies, Palo Alto, CA, USA). MicroRNA microarraying of each pooled sample was performed by the same method as that described above.

RNA isolation from serum samples. One hundred microliters of serum was thawed on ice and then transferred to a tube containing $150 \mu \mathrm{l}$ of nuclease-free water. The samples were each added to $750 \mu \mathrm{l}$ of TRIzol LS reagent (Invitrogen), followed by homogenisation by pipetting up and down several times. To allow for normalisation of sample-to-sample variation, $25 \mathrm{fmol}$ of synthetic C. elegans miRNA cel-miR-39 ( $5 \mu \mathrm{l}$ of $5 \mathrm{~nm}$ miRNA mimic) (Qiagen, Hilden, Germany) was added to the denatured samples. Total RNA in serum samples was then extracted according to the manufacturer's protocol.

Real-time quantitative RT- PCR (qRT-PCR) of miRNAs. The levels of miRNAs in serum samples were determined using TaqMan MicroRNA Assays (Applied Biosystems, Foster City, CA, USA). TaqMan miRNA reverse transcription was performed with miRNA-specific stem-loop primers. The amplification was carried out by denaturation at $95^{\circ} \mathrm{C}$ for $10 \mathrm{~min}$, followed by 45 cycles of $95^{\circ} \mathrm{C}$ for $15 \mathrm{~s}$ and $58^{\circ} \mathrm{C}$ for $60 \mathrm{~s}$. Each reaction was performed in triplicate. Spiked-in cel-miR-39 was analysed as a normalisation control and relative expression was calculated using the $2^{-\Delta \Delta \mathrm{Ct}}$ method (Livak and Schmittgen, 2001).

Statistical analysis. $\mathrm{R}$ statistical software ( $\mathrm{R}$ Foundation for Statistical Computing, Vienna, Austria) was utilised to calculate correlation coefficients in regression analyses. Fisher's exact test, Student's $t$-test and Mann-Whitney $U$-test were also performed using R. Statistical significance was defined as $P<0.05$. The sensitivity, specificity and cutoff value were calculated according to standard formulas. The receiver-operating characteristic (ROC) curve and area under the curve (AUC) were established to discriminate mice with and without DGC.

\section{RESULTS}

Comparison of miRNA expression profiles of mouse and human primary GC. We analysed the miRNA profiles of primary DGC in DCKO mice using Mouse miRNA Oligo chip ver.16. To compare the miRNA expression profiles of mouse and human GC, we investigated the expression levels of miRNAs that were found to be 
A

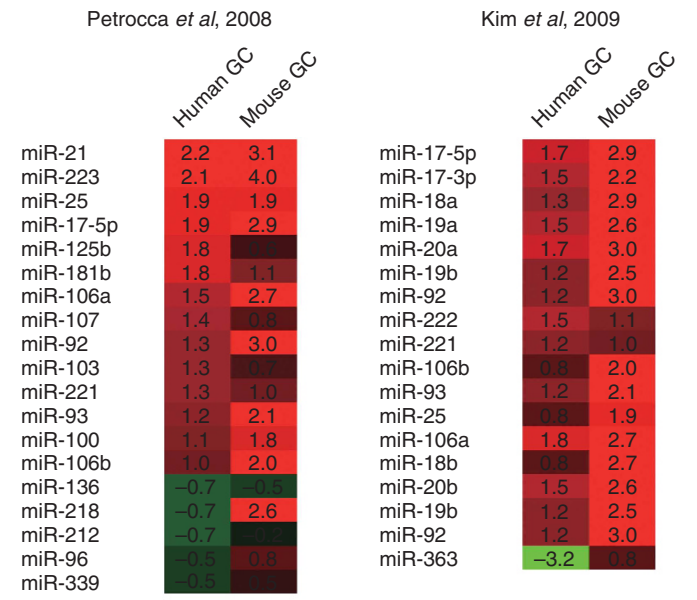

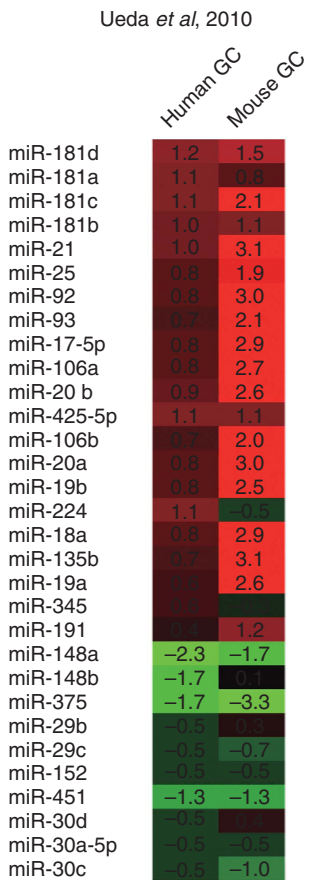

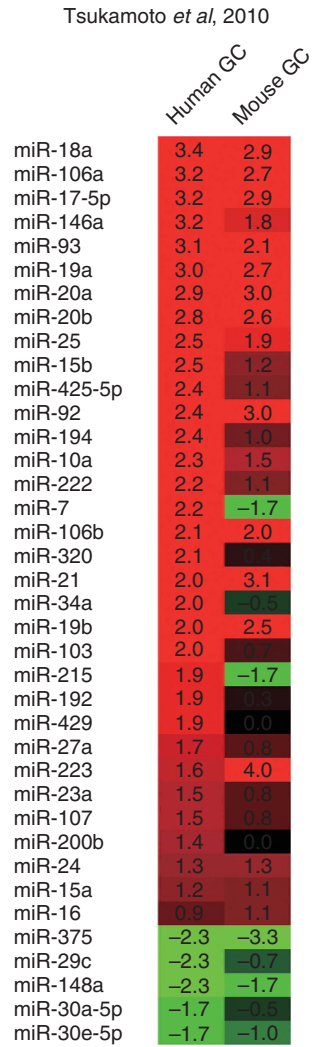

B

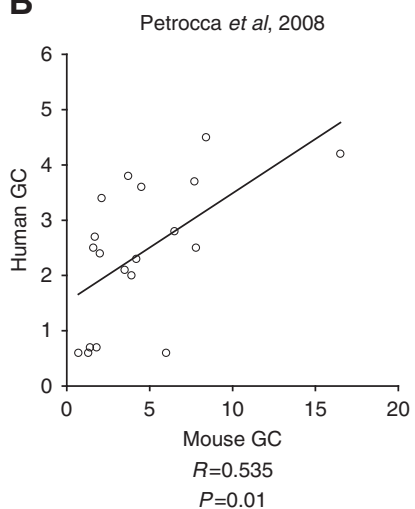

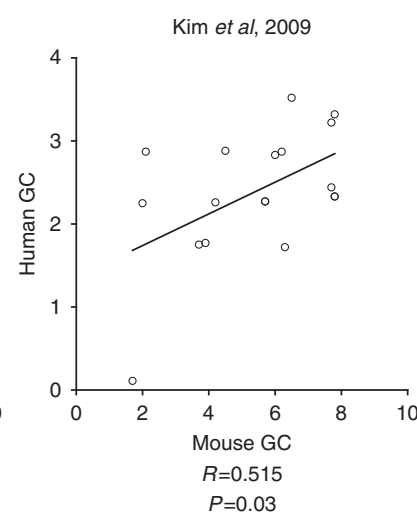
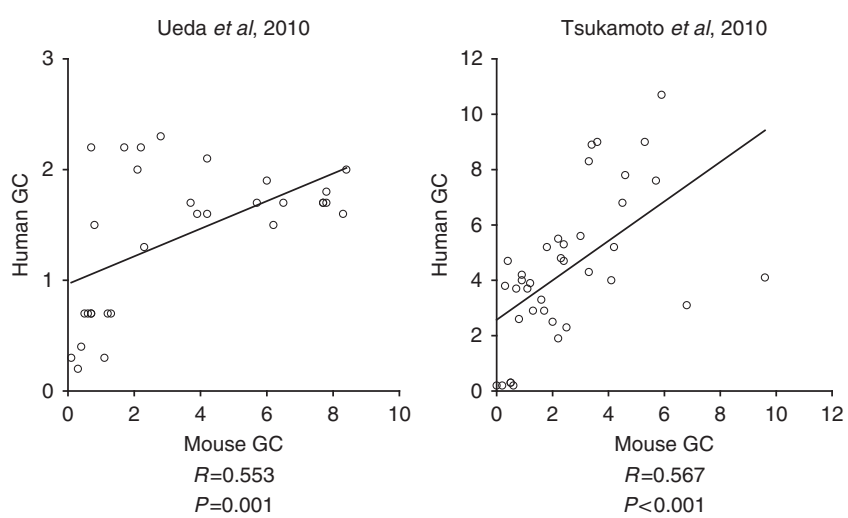

Figure 1. Heat maps (A) and dot plots (B) of miRNA expression profiles in mouse and human primary GC using homologous miRNAs reported by Petrocca et al (2008), Kim et al (2009), Ueda et al (2010) and Tsukamoto et al (2010), respectively. The expression levels of miRNAs are represented by $\log 2$ ratios and fold-changes as to normal gastric tissues from control mice or humans in heat maps and dot plots, respectively.

significantly upregulated or downregulated in human primary GC in four published studies (Petrocca et al, 2008; Kim et al, 2009; Ueda et al, 2010; Tsukamoto et al, 2010). Out of them, we selected miRNAs that were detected in Mouse miRNA Oligo chip ver.16, and visualised the miRNA expression patterns of mouse and human GC by means of heat maps (Figure 1a) and dot plots (Figure 1b). These comparative analyses demonstrated that DGC in DCKO mice was very similar to human GC in their miRNA signatures $(P=0.01,0.03,0.001$ and $P<0.001$, respectively).

Ueda et al (2010) have reported that eight and four miRNAs were overexpressed in human DGC and IGC, respectively. Notably, seven (miR-100, miR-125b, miR-199a, miR-99a, miR-143, miR145 and miR-133a) of eight miRNAs upregulated in human DGC were highly expressed in mouse DGC, while none of four miRNAs upregulated in human IGC were $(P=0.01$; Fisher's exact test). Overall, microarray analyses of primary GC revealed that DCKO mice could recapitulate human DGC at the miRNA level.
Correlation of miRNA signatures of mouse tissue and serum samples. We analysed the miRNA characteristics of pooled sera from DCKO and control mice, primary GC and LN metastases of DCKO mice, and normal stomach and LN of control mice using miRNA microarrays. We selected 774 miRNAs that were detected in all of our microarray assays, and performed regression analyses of them (Figure 2). The correlation coefficients of miRNA expression patterns between normal gastric mucosae and primary GC, and normal LN and metastatic GC were high, that is, 0.933 and 0.926 , respectively (Figure $2 \mathrm{a}$ ), indicating that the differences between normal and cancer tissues are small. The correlation coefficient between primary and metastatic GC in miRNA profile, 0.973 , was higher than the other two coefficients $(P<0.001$ for both; Student's $t$-test), suggesting that primary and metastatic GC exhibit similar characteristics as to miRNA expression.

Figure $2 \mathrm{~b}$ shows that the miRNA patterns in sera were not closely associated with those in gastric tissue samples in DCKO 
A $P<0.001$

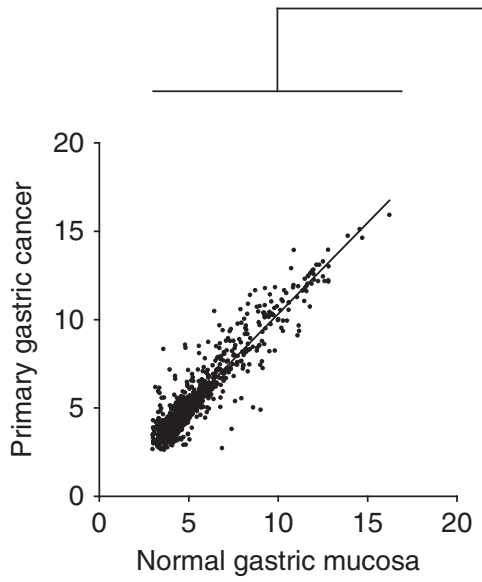

$R=0.933$

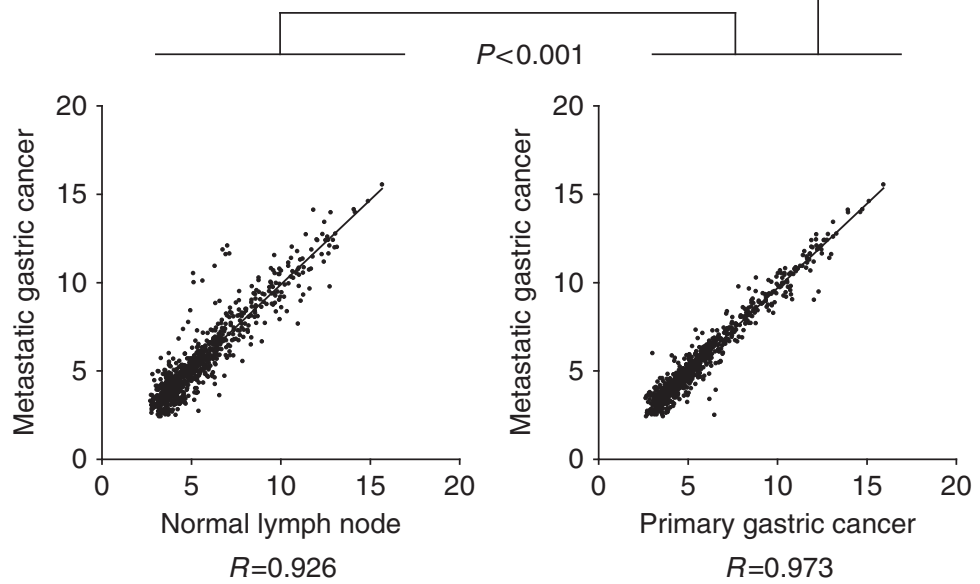

B

$P<0.001$
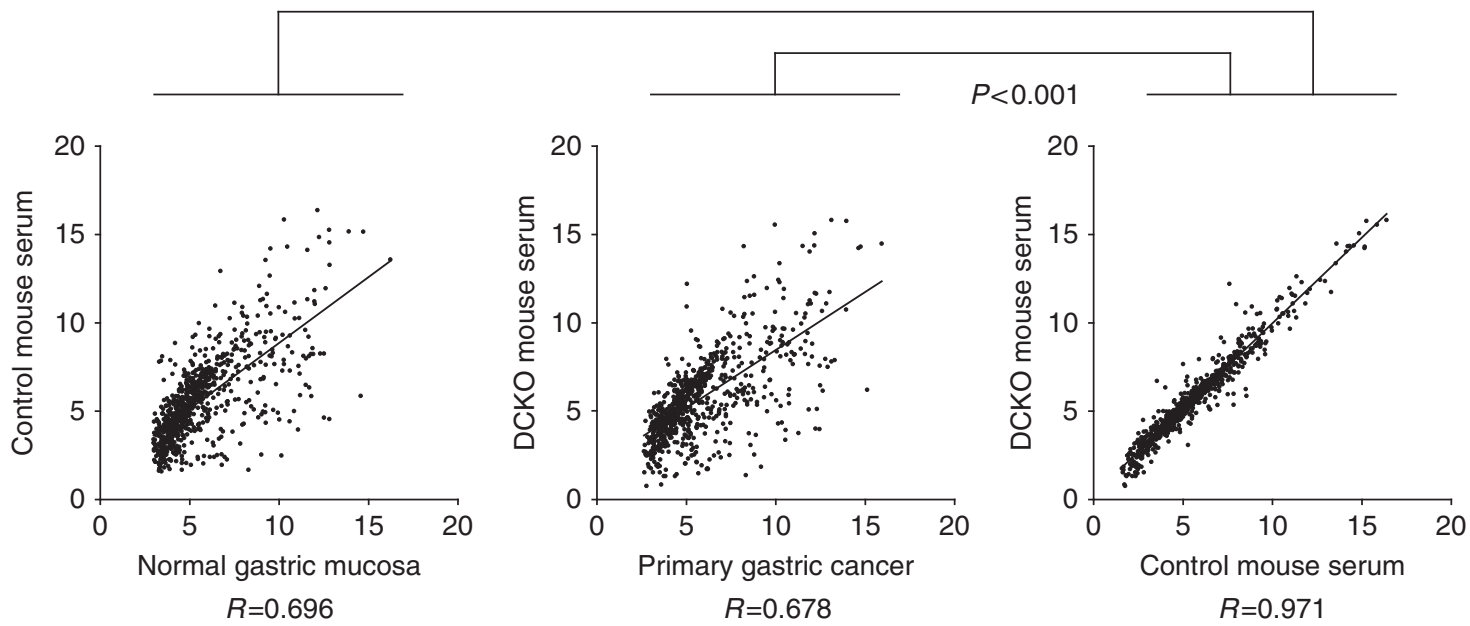

Figure 2. Dot plots of miRNA signatures in mouse normal and cancer tissue specimens (A), and in mouse tissue and serum samples (B).

mice or littermate controls $(R=0.678$ and 0.696 , respectively). Sera of cancer-positive and -negative mice exhibited higher miRNA expression similarity $(R=0.971)$ than pairs of tissue and serum samples in the two groups of mice $(P<0.001$ for both; Student's $t$-test), indicating that only some miRNAs overexpressed in cancer tissues could be released into the serum.

Identification of DGC-related miRNAs in sera using microarrays. We employed a strategy including screening by means of miRNA microarrays using pooled samples and validation by TaqMan qRT-PCR for individual mouse samples at different ages (Figure 3a). We initially searched for candidate miRNAs by comparing the miRNA profiles of DCKO and control mice in three types of samples: (1) DCKO vs control mouse sera, (2) primary DGC $v s$ normal stomach tissues and (3) metastatic DGC $v s$ normal stomach tissues. As shown in Figure $3 \mathrm{~b}$ and Supplementary Table S2, the microarray analyses revealed 27 upregulated miRNAs in the sera and GC of DCKO mice compared with in controls, of which 18 were increased in LN metastasis specimens as well. In addition, 75 miRNAs that were significantly upregulated only in the sera were also identified. We selected candidate miRNAs that satisfied three criteria: (1) the level in DCKO mouse serum was $>1.5$-fold higher than that in controls; (2) the global normalisation value was $>100$ in DCKO mouse serum, indicating easily detectable levels of miRNAs; and (3) the identified miRNAs were coincidently upregulated in both sera and DGC tissues.
Candidate miRNA selection. Among the 27 and 75 candidate miRNAs identified on microarray analyses, 5 were selected for further validation based on previous papers, in which these miRNAs were reported to have important functions in carcinogenesis. We chose miR-103 and miR-107, which belong to the same family, because these two miRNAs were simultaneously upregulated in both sera and DGC tissues, but not significantly in LN metastasis tissues. The higher expression of the miR-103/107 family has been reported not only in GC (Tsukamoto et al, 2010) but also in other cancers such as pancreatic (Roldo et al, 2006), breast (Martello et al, 2010) and prostate (Lodes et al, 2009) cancers. In addition, miR-194 and miR-210 were selected from the group of miRNAs increased in sera, DGC and LN metastasis tissues. The miR-194 level was considerably high in sera (6.3-fold) and LN metastases (1.8-fold). MiR-210 has been suggested to be a serum biomarker for diffuse large B-cell lymphoma and pancreatic cancer (Cortez et al, 2011).

Interestingly, five members of the miR-290-295 cluster, miR-291b-5p, miR-291a-5p, miR-290-5p, miR-294* and miR292-5p, exhibited 5.54-, 4.81-, 3.26-, 2.52- and 1.56-fold higher expression levels in DCKO mouse sera, respectively. The miR-290295 cluster codes a family of miRNAs that was identified as cellcycle controllers via targeting of the cell-cycle regulator genes, Wee1 and Fbxl5 (Lichner et al, 2011). A redundancy of this miRNA family in diseased mouse sera may reflect their critical functions and ability to serve as serum biomarkers. Thus, we 
A
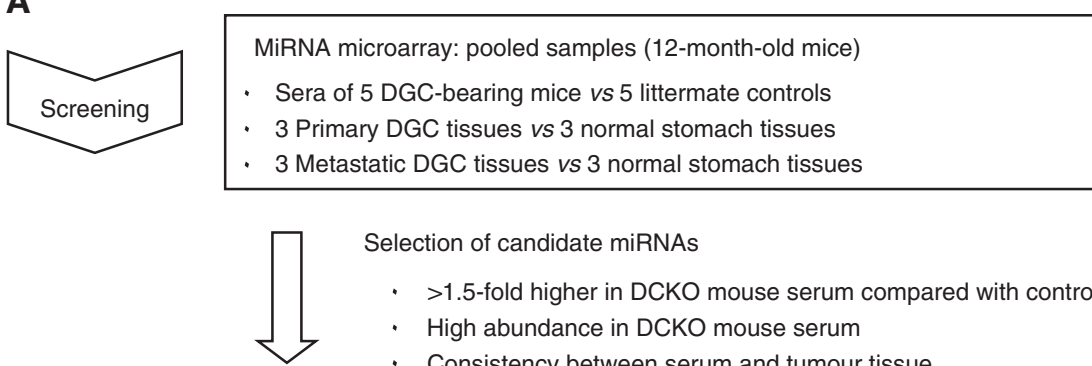

Selection of candidate miRNAs

- $\quad>1.5$-fold higher in DCKO mouse serum compared with control serum

- High abundance in DCKO mouse serum

- Consistency between serum and tumour tissue
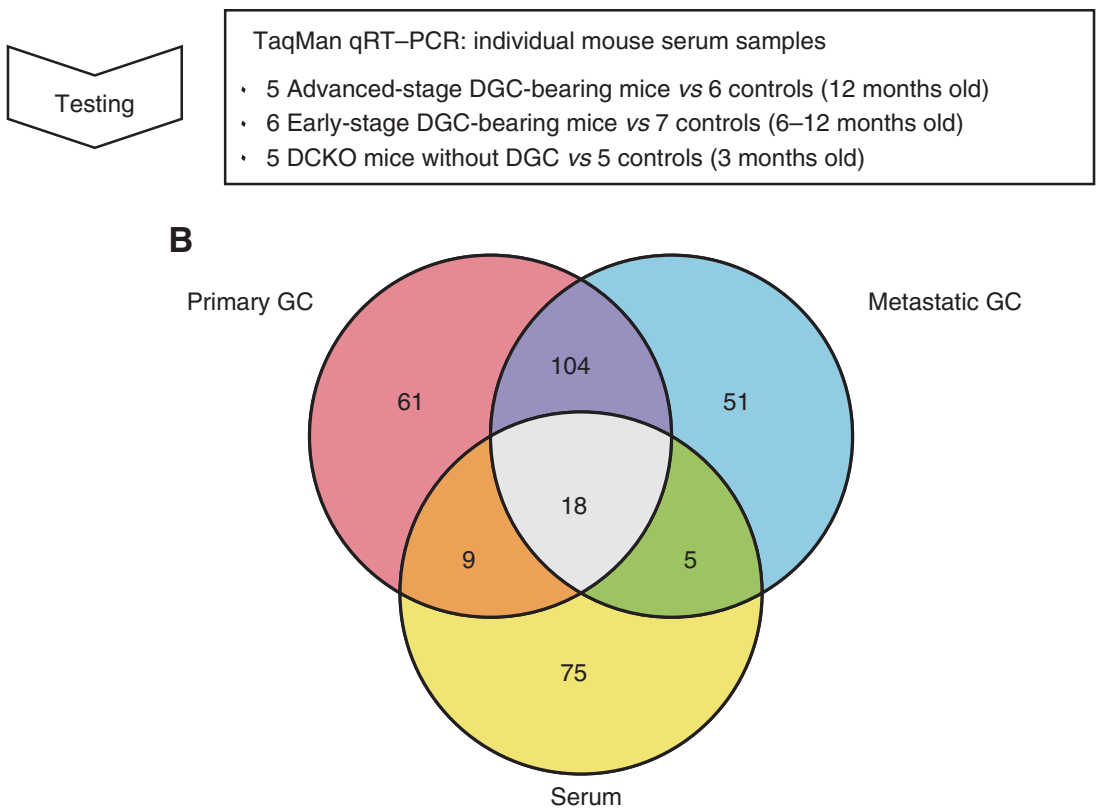

Figure 3. (A) Overview of miRNA analyses of DCKO mouse tissue and serum samples. (B) The three-way Venn diagram showing the numbers of upregulated miRNAs in DCKO mouse samples overlapping in sera, DGC tissues and lymphatic metastasis tissues.

picked miR-291b-5p as a representative for this cluster for the next validation. Totally, we selected five miRNAs, that is, miR-103, miR107, miR-194, miR-210 and miR-291b-5p, as candidate biomarkers.

Validation of serum miRNAs by $\mathrm{qRT}$-PCR in mice at different cancer stages. To evaluate the accuracy of microarray data, the levels of the five selected miRNAs in serum samples were individually assessed by TaqMan qRT-PCR. First, we examined the miRNA levels in 12-month-old DCKO mice with advancedstage DGC $(n=5)$ and age-matched controls $(n=6)$ (Figure $4 \mathrm{a})$. Four of the five miRNAs, miR-103, miR-107, miR-194 and miR210, showed significant elevation in DCKO mouse sera at $P=0.045,0.004,0.004$ and 0.030 , respectively (Mann-Whitney $U$-test). These results indicate that the microarray analysis data were mostly reliable. Secondly, these five miRNAs were further quantified in 6-12-month-old DCKO mice with histologically proven intramucosal DGC $(n=6)$ and age-matched littermates $(n=7)$ (Figure $4 \mathrm{~b}$ ). Similar to the results for mice with advanced DGC, the levels of miR-103, miR-107, miR-194 and miR-210 were significantly increased in DCKO mouse sera $(P=0.014,0.022$, 0.014 and 0.022 , respectively), suggesting the potential of these four miRNAs for identifying DGC at an early stage.

It was questionable whether upregulation of miR-103, miR-107, miR-194 and miR-210 in mice with early- and advanced-stage DGC resulted from inactivation of E-cadherin and p53 in the cells-oforigin, or was induced by the process of malignant transformation. We thus investigated whether or not the levels of the candidate miRNAs were changed in mice at 3 months of age $(n=5)$, in which DGC had not been detected. As shown in Figure 4c, no significant elevation of any miRNA, miR-103, miR-107, miR-194, miR-210 or miR-291b-5p, was found in DCKO mouse sera $(P=0.421,0.690$, $0.548,0.548$ and 0.151 , respectively). This evidence suggested that upregulation of miR-103, miR-107, miR-194 and miR-210 in mice with early- and advanced-stage DGC was triggered by aberrant processes during the cancer development.

Profiles of serum miRNA levels during DGC development. The levels of the four miRNAs were examined in DCKO mice at different time points during DGC progression. The medians of their levels in both DCKO and control mouse sera were plotted at 3 months of age (no DGC), 6-12 months of age (early-stage DGC), and 12 months of age (advanced-stage DGC). Understanding the trend of candidate miRNA levels could provide an insight as to biomarker determination. Ideal biomarkers would not only be able to discriminate DGC patients from healthy ones, but also indicate the clinical stage. As shown in Figure 5, the levels of miR-103, miR-107 and miR-194 in control mouse sera were relatively constant, but not that of miR-210, whose level was significantly reduced in 12month-old mice $(P=0.045)$. On the other hand, upregulation of the miR-103 and miR-194 levels in DCKO mouse sera occurred in a stepwise manner during the progression from normal to early-stage DGC and finally to advanced-stage DGC, suggesting both of them are very suitable biomarkers for DGC diagnosis.

Although the level of miR-107 in DCKO mouse sera was slightly decreased at the advanced stage, its level was still significantly higher than that in the no DGC group $(P=0.008)$. Therefore, miR-107 could be a biomarker of choice for differentiating DGC-bearing mice from normal ones. However, the level of 
A
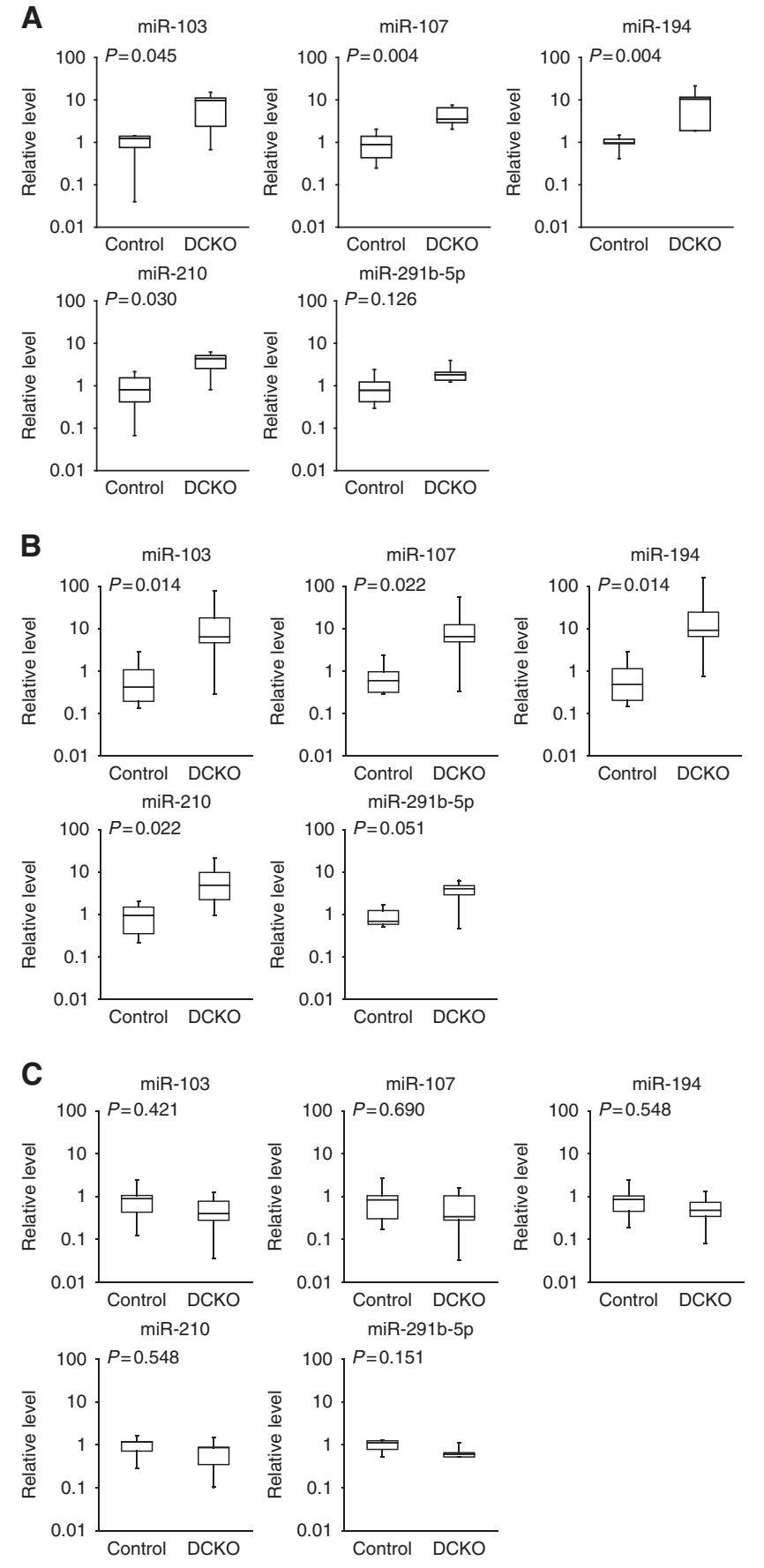

Figure 4. (A) Comparison of serum miRNA levels between DCKO $(n=5)$ and control $(n=6)$ mice at 12 months of age. All DCKO mice had developed advanced-stage DGC. (B) Comparison of serum miRNA levels between DCKO mice with early-stage DGC $(n=6)$ and control mice $(n=7)$ at $6-12$ months of age. (C) Comparison of serum miRNA levels between DCKO $(n=5)$ and control $(n=5)$ mice at 3 months of age. Double conditional knockout mice had not developed GC yet at this age. The levels of miRNAs in all the samples were determined by qRT-PCR analyses.

miR-210 did not show a significant difference between 12-monthold DCKO mice with advanced-stage DGC and 3-month-old DCKO mice without cancer, leading to the idea that miR-210 may be an improper indicator if DGC is already advanced.

Risk assessment of candidate miRNAs in discriminating DGC from non-cancer cases. An ROC curve was plotted to identify cutoff values for miR-103, miR-107, miR-194 and miR-210 that could be used to distinguish DGC-positive cases from healthy controls (Figure 6). ROC curve analyses showed that at the cutoff level of 2.58, miR-103 exhibited $81.8 \%$ sensitivity and $95.7 \%$ specificity with an AUC of 0.881 . At the cutoff level of 2.11 , miR107 exhibited $90.9 \%$ sensitivity and $95.7 \%$ specificity with an AUC of 0.909 . At the cutoff level of 3.36, miR-194 exhibited $90.9 \%$ sensitivity and $95.7 \%$ specificity with an AUC of 0.925 . At the cutoff level of 2.29 , miR-210 exhibited $72.7 \%$ sensitivity and $87.0 \%$ specificity with an AUC of 0.846 .

\section{DISCUSSION}

A mouse model of human cancer could be an efficient means of discovering diagnostic markers not only because the genetic alterations associated with human tumours can be engineered in mice, but also because mouse and human cancers can exhibit similar molecular signatures. We demonstrated that primary GC and LN metastases induced in DCKO mice resembled human GC in their miRNA expression profiles. We used this animal model of DGC to identify the circulating miRNAs that can serve as non-invasive biomarkers for DGC diagnosis. As a result of microarray analyses, five miRNAs, miR-103, miR-107, miR-194, miR-210 and miR-291b$5 p$, that were increased in sera from DCKO mice with DGC, were selected as candidate biomarkers. We validated that the levels of four of these five circulating miRNAs, that is, miR-103, miR-107, miR-194 and miR-210, were upregulated in sera of mice with both early- and advanced-stage DGC compared with in controls.

Several studies have revealed subsets of miRNAs whose expression levels are upregulated or downregulated in human GC (Petrocca et al, 2008; Kim et al, 2009; Tsukamoto et al, 2010; Ueda et al, 2010). They exhibited similar miRNA signatures, such as overexpression of miR-21, miR-17-92 and miR-106b-25 clusters, consistent with our finding that these miRNAs were highly expressed in primary DGC of DCKO mice on microarray analyses. In contrast, although other research groups have identified some miRNAs differentially expressed in sera or plasma of human GC patients as biomarkers, there has been a conflict as to their levels in those studies (Tsujiura et al, 2010; Liu et al, 2011; Konishi et al, 2012; Liu et al, 2012; Song et al, 2012). So far, no overlap of miRNA biomarkers for GC detection has been reported. The inconsistency of these results could be explained by many factors, particularly variations in histopathological types and experimental methods. The GC samples used in previous studies comprised combination of both histological subtypes (intestinaland diffuse-types).

On the other hand, our study only involved DGC-associated samples, leading to the difference in discovered miRNAs compared with those in human GC samples. However, we found that five miRNAs had the corresponding probes in our mouse miRNA microarray data among the 11 upregulated circulating miRNAs in human GC cases reported by Liu et al (2012), and that four (miR$150^{\star}, \mathrm{miR}-371-5 \mathrm{p}, \mathrm{miR}-187^{\star}$ and miR-378) of these five miRNAs showed similar upregulation in sera of DCKO mice. These results suggest that the changes in serum miRNA levels in DCKO mice could be comparable to those in human GC patients.

Furthermore, there has been another study discovering miRNA biomarkers using a mouse model of prostate cancer (Selth et al, 2012). They performed comprehensive and quantitative analyses of serum miRNAs of the transgenic mice in the same manner as we did in this work, and have demonstrated that some serum miRNAs identified in diseased mice are shared between human and mouse prostate cancers. Therefore, although further validation with a large cohort of human samples and a prospective study are required, four miRNAs identified in this study, miR-103, miR-107, 

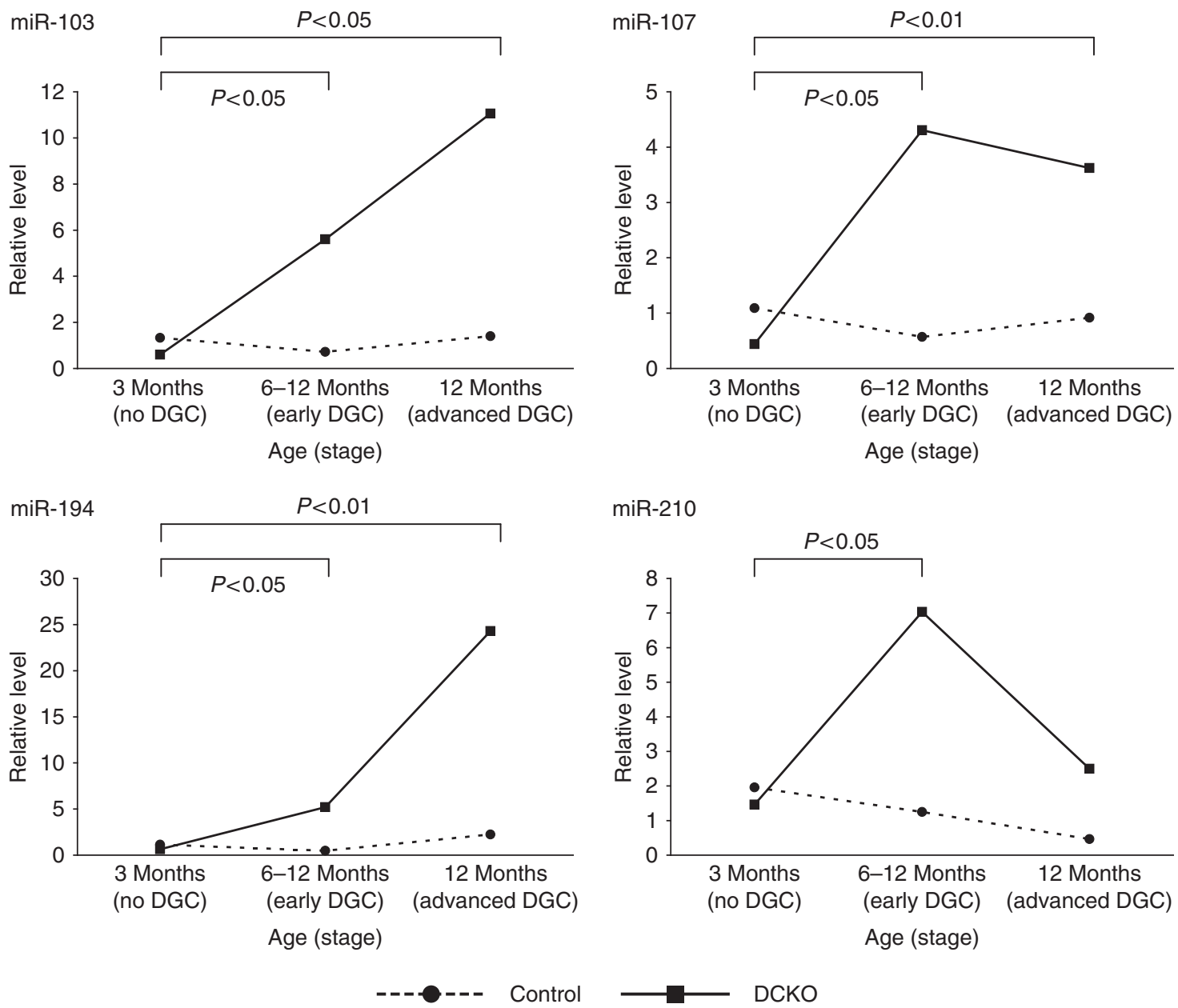

Figure 5. Time course of serum miRNA levels during DGC development. The medians of the relative levels of miR-103, miR-107, miR-194 and miR-210 in DCKO mouse sera were plotted at 3 (without DGC), 6-12 (with early-stage DGC) and 12 months of age (with advanced-stage DGC). The shape of the line shows the expression trend of the miRNAs during DGC progression.
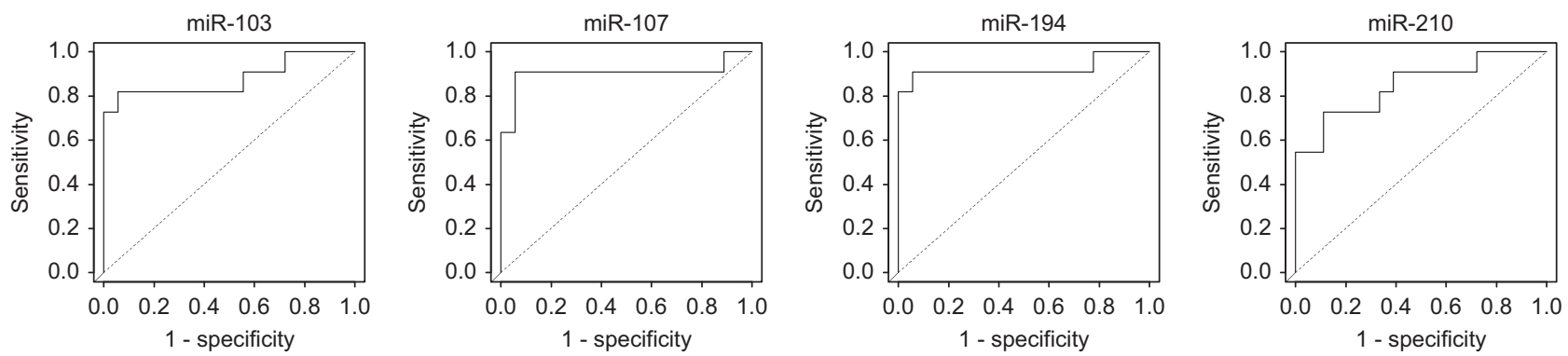

Figure 6. Receiver-operating characteristic curve analyses of the serum miR-103, miR-107, miR-194 and miR-210 levels to discriminate mice with DGC ( $n=11$; not including 3-month-old DCKO mice) from healthy ones $(n=18)$.

miR-194 and miR-210, could be promising as effective serum markers for DGC diagnosis and screening.

The activation of miR-103 and miR-107 in GC is consistent with a previous study that involved human tissue samples (Tsukamoto et al, 2010). There is evidence that the miR-103/107 family induces the epithelial-to-mesenchymal transition (EMT) in breast cancer (Martello et al, 2010). Taken together with our finding that EMT could have crucial roles in diffuse-type carcinogenesis in DCKO mice (Shimada et al, 2012), this miRNA family might be associated with the development and/or progression of the malignancy. Further functional studies on this miRNA family to determine their interactions with tumour-suppressor genes are required.
Our data showed that the miR-194 level was upregulated in all types of samples obtained from DCKO mice with DGC, that is, serum, DGC tissues and LN metastasis tissues. In a former study, Tsukamoto et al (2010) demonstrated that the elevation of miR-194 was as high as 5.16-fold in human GC tissues. Moreover, miR-194 has been reported to be overexpressed in oesophageal cancer (Song and Meltzer, 2012), and to be involved in the carcinogenesis in pancreatic ductal adenocarcinomas (Mees et al, 2010). It has been proved that miR-194 regulates metastasis formation through targeting of the metastasis-suppressor gene, EP300 (Mees et al, 2010). However, the oncogenic role of miR-194 in DGC metastasis has not been determined. 
In addition to for DGC, a high level of miR-210 has been supposed to be a diagnostic biomarker for other malignancies, that is, diffuse large B-cell lymphoma (Lawrie et al, 2008) and pancreatic cancer (Wang et al, 2009; Ho et al, 2010), and a prognostic marker for breast cancer (Camps et al, 2008). Two reports have mentioned that miR-210 was directly regulated by HIF1A and therefore induced by hypoxia, a common feature of solid tumours (Crosby et al, 2009; Mathew and Simon, 2009). Although the miR-210 level in DCKO mouse sera with advanced-stage DGC was not significantly different from that in DCKO mice without cancer, comparison of 12-monthand 3-month-old control mice also showed the similar trend that the miR-210 level had significantly decreased by 12 months of age $(P=0.045)$. This reduction in the miR-210 level in 12 -month-old mice for both DCKO and control mice might be dependent on age.

In summary, we have demonstrated that a mouse model of human GC provided helpful materials for discovering circulating miRNAs relevant to the clinical stage of DGC. We found that four miRNAs, miR-103, miR-107, miR-194 and miR-210, were specifically upregulated in DGC-positive mouse sera, and suggested they are novel non-invasive biomarkers for the early detection of DGC. Furthermore, the elevation of miR-103 and miR-194 occurred during the entire progression from the noncancer status to the advanced-stage, implying their ability as biomarkers that can be used to determine the DGC stage.

\section{ACKNOWLEDGEMENTS}

This study was supported by a Grant-in-Aid for Scientific Research (B), No. 2330342, and by the A3 Foresight Program from JSPS.

\section{CONFLICT OF INTEREST}

The authors declare no conflict of interest.

\section{REFERENCES}

Arroyo JD, Chevillet JR, Kroh EM, Ruf IK, Pritchard CC, Gibson DF, Mitchell PS, Bennett CF, Pogosova-Agadjanyan EL, Stirewalt DL, Tait JF, Tewari M (2011) Argonaute 2 complexes carry a population of circulating microRNAs independent of vesicles in human plasma. Proc Natl Acad Sci USA 108: 5003-5008.

Camps C, Buffa FM, Colella S, Moore J, Sotiriou C, Sheldon H, Harris AL, Gleadle JM, Ragoussis J (2008) hsa-miR-210 is induced by hypoxia and is an independent prognostic factor in breast cancer. Clin Cancer Res 14: $1340-1348$.

Cortez MA, Bueso-Ramos C, Ferdin J, Lopez-Berestein G, Sood AK, Calin GA (2011) MicroRNAs in body fluids-the mix of hormones and biomarkers. Nat Rev Clin Oncol 8: 467-477.

Crosby ME, Kulshreshtha R, Ivan M, Glazer PM (2009) MicroRNA regulation of DNA repair gene expression in hypoxic stress. Cancer Res 69 1221-1229.

Ferlay J, Shin HR, Bray F, Forman D, Mathers C, Parkin DM (2010) Estimates of worldwide burden of cancer in 2008: GLOBOCAN 2008. Int J Cancer 127: 2893-2917.

Ho AS, Huang X, Cao H, Christman-Skieller C, Bennewith K, Le QT, Koong AC (2010) Circulating miR-210 as a novel hypoxia marker in pancreatic cancer. Transl Oncol 3: 109-113.

Japanese Gastric Cancer Association (1998) Japanese Classification of Gastric Carcinoma - 2nd English Edition. Gastric Cancer 1: 10-24.

Kim YK, Yu J, Han TS, Park SY, Namkoong B, Kim DH, Hur K, Yoo MW, Lee HJ, Yang HK, Kim VN (2009) Functional links between clustered microRNAs: suppression of cell-cycle inhibitors by microRNA clusters in gastric cancer. Nucleic Acids Res 37: 1672-1681.
Konishi H, Ichikawa D, Komatsu S, Shiozaki A, Tsujiura M, Takeshita H, Morimura R, Nagata H, Arita T, Kawaguchi T, Hirashima S, Fujiwara H, Okamoto K, Otsuji E (2012) Detection of gastric cancer-associated microRNAs on microRNA microarray comparing pre- and post-operative plasma. Br J Cancer 106: 740-747.

Kuick R, Misek DE, Monsma DJ, Webb CP, Wang H, Peterson KJ, Pisano M, Omenn GS, Hanash SM (2007) Discovery of cancer biomarkers through the use of mouse models. Cancer Lett 249: 40-48.

Laurén P (1965) The two histological main types of gastric carcinoma: diffuse and so-called intestinal-type carcinoma. An attempt at a histo-clinical classification. Acta Pathol Microbiol Scand 64: 31-49.

Lawrie CH, Gal S, Dunlop HM, Pushkaran B, Liggins AP, Pulford K, Banham AH, Pezzella F, Boultwood J, Wainscoat JS, Hatton CS, Harris AL (2008) Detection of elevated levels of tumour-associated microRNAs in serum of patients with diffuse large B-cell lymphoma. Br J Haematol 141: 672-675.

Lichner Z, Páll E, Kerekes A, Pállinger E, Maraghechi P, Bosze Z, Gócza E (2011) The miR-290-295 cluster promotes pluripotency maintenance by regulating cell cycle phase distribution in mouse embryonic stem cells. Differentiation 81: 11-24.

Liu H, Zhu L, Liu B, Yang L, Meng X, Zhang W, Ma Y, Xiao H (2012) Genome-wide microRNA profiles identify miR-378 as a serum biomarker for early detection of gastric cancer. Cancer Lett 316: 196-203.

Liu R, Zhang C, Hu Z, Li G, Wang C, Yang C, Huang D, Chen X, Zhang H, Zhuang R, Deng T, Liu H, Yin J, Wang S, Zen K, Ba Y, Zhang CY (2011) A five-microRNA signature identified from genome-wide serum microRNA expression profiling serves as a fingerprint for gastric cancer diagnosis. Eur J Cancer 47: 784-791.

Livak KJ, Schmittgen TD (2001) Analysis of relative gene expression data using real-time quantitative PCR and the 2(-Delta Delta $\mathrm{C}(\mathrm{T})$ ) Method. Methods 25: 402-408.

Lodes MJ, Caraballo M, Suciu D, Munro S, Kumar A, Anderson B (2009) Detection of cancer with serum miRNAs on an oligonucleotide microarray. PLoS One 4: e6229.

Martello G, Rosato A, Ferrari F, Manfrin A, Cordenonsi M, Dupont S, Enzo E, Guzzardo V, Rondina M, Spruce T, Parenti AR, Daidone MG, Bicciato S, Piccolo S (2010) A microRNA targeting dicer for metastasis control. Cell 141: 1195-1207.

Mathew LK, Simon MC (2009) mir-210: a sensor for hypoxic stress during tumorigenesis. Mol Cell 35: 737-738.

Mees ST, Mardin WA, Wendel C, Baeumer N, Willscher E, Senninger N, Schleicher C, Colombo-Benkmann M, Haier J (2010) EP300-a miRNAregulated metastasis suppressor gene in ductal adenocarcinomas of the pancreas. Int J Cancer 126: 114-124.

Petrocca F, Visone R, Onelli MR, Shah MH, Nicoloso MS, de Martino I, Iliopoulos D, Pilozzi E, Liu CG, Negrini M, Cavazzini L, Volinia S, Alder H, Ruco LP, Baldassarre G, Croce CM, Vecchione A (2008) E2F1regulated microRNAs impair TGFbeta-dependent cell-cycle arrest and apoptosis in gastric cancer. Cancer Cell 13: 272-286.

Roldo C, Missiaglia E, Hagan JP, Falconi M, Capelli P, Bersani S, Calin GA, Volinia S, Liu CG, Scarpa A, Croce CM (2006) MicroRNA expression abnormalities in pancreatic endocrine and acinar tumors are associated with distinctive pathologic features and clinical behavior. J Clin Oncol 24: 4677-4684.

Selth LA, Townley S, Gillis JL, Ochnik AM, Murti K, Macfarlane RJ, Chi KN, Marshall VR, Tilley WD, Butler LM (2012) Discovery of circulating microRNAs associated with human prostate cancer using a mouse model of disease. Int J Cancer 131: 652-661.

Shimada S, Mimata A, Sekine M, Mogushi K, Akiyama Y, Fukamachi H, Jonkers J, Tanaka H, Eishi Y, Yuasa Y (2012) Synergistic tumour suppressor activity of E-cadherin and p53 in a conditional mouse model for metastatic diffuse-type gastric cancer. Gut 61: 344-353.

Song JH, Meltzer SJ (2012) MicroRNAs in pathogenesis, diagnosis, and treatment of gastroesophageal cancers. Gastroenterology 143: 35-47.

Song MY, Pan KF, Su HJ, Zhang L, Ma JL, Li JY, Yuasa Y, Kang D, Kim YS, You WC (2012) Identification of serum microRNAs as novel non-invasive biomarkers for early detection of gastric cancer. PLoS One 7: e33608.

Tsujiura M, Ichikawa D, Komatsu S, Shiozaki A, Takeshita H, Kosuga T, Konishi H, Morimura R, Deguchi K, Fujiwara H, Okamoto K, Otsuji E (2010) Circulating microRNAs in plasma of patients with gastric cancers. Br J Cancer 102: 1174-1179.

Tsukamoto Y, Nakada C, Noguchi T, Tanigawa M, Nguyen LT, Uchida T, Hijiya N, Matsuura K, Fujioka T, Seto M, Moriyama M (2010) 
MicroRNA-375 is downregulated in gastric carcinomas and regulates cell survival by targeting PDK1 and 14-3-3zeta. Cancer Res 70: 2339-2349.

Ueda T, Volinia S, Okumura H, Shimizu M, Taccioli C, Rossi S, Alder H, Liu CG, Oue N, Yasui W, Yoshida K, Sasaki H, Nomura S, Seto Y, Kaminishi M, Calin GA, Croce CM (2010) Relation between microRNA expression and progression and prognosis of gastric cancer: a microRNA expression analysis. Lancet Oncol 11: 136-146.

Valadi H, Ekström K, Bossios A, Sjöstrand M, Lee JJ, Lötvall JO (2007) Exosome-mediated transfer of mRNAs and microRNAs is a novel mechanism of genetic exchange between cells. Nat Cell Biol 9: 654-659.

Vickers KC, Palmisano BT, Shoucri BM, Shamburek RD, Remaley AT (2011) MicroRNAs are transported in plasma and delivered to recipient cells by high-density lipoproteins. Nat Cell Biol 13: 423-433.
Wang J, Chen J, Chang P, LeBlanc A, Li D, Abbruzzesse JL, Frazier ML, Killary AM, Sen S (2009) MicroRNAs in plasma of pancreatic ductal adenocarcinoma patients as novel blood-based biomarkers of disease. Cancer Prev Res 2: 807-813.

Yuasa Y (2003) Control of gut differentiation and intestinal-type gastric carcinogenesis. Nat Rev Cancer 3: 592-600.

This work is published under the standard license to publish agreement. After 12 months the work will become freely available and the license terms will switch to a Creative Commons AttributionNonCommercial-Share Alike 3.0 Unported License.

Supplementary Information accompanies this paper on British Journal of Cancer website (http://www.nature.com/bjc) 